\title{
Validity of currently used cutoff values of body mass index as a measure of obesity in Sri Lankan children
}

\author{
V P Wickramasinghe ${ }^{1}$, S P Lamabadusuriya ${ }^{1}$, G J Cleghorn ${ }^{2}$ and P S W Davies ${ }^{2}$
}

(Index words: children, body composition, fat mass, BMI)

\begin{abstract}
Objective The aim of the study was to determine the reliability of body mass index based (BMI) cutoff values in diagnosing obesity among Sri Lankan children.
\end{abstract}

Methods Height, weight, waist circumference (WC) and hip circumference $(\mathrm{HC})$ in 282 children were measured. Total body water was determined by deuterium dilution and fat mass (FM) derived using age and gender specific constants. A percentage $\mathrm{FM}$ of $30 \%$ for girls and $25 \%$ for boys were considered as cutoff levels for obesity.

Results Two hundred and eighty two children (M/F: 158/124) were studied and 99 (80\%) girls and 72 (45.5\%) boys were obese based on \% body fat. Eight (6.4\%) girls and nine (5.7\%) boys were obese based on International Obesity Task Force (IOTF) cutoff values. Percentage FM and WC centile charts were able to diagnose a significant proportion of children as true obese children. The FM and BMI were closely associated in both girls $(r=0.82$, $p<0.001)$ and boys $(r=0.87, p<0.001)$. Percentage FM and BMI had a very low but significant association; girls $(r=0.32, p<0.001)$ and boys $(r=0.68, p<0.001)$. FM had a significant association with $\mathrm{WC}$ and $\mathrm{HC}$. BMI based cutoff values had a specificity of $100 \%$ but a very low sensitivity, varying between $8 \%$ and $23.6 \%$.

${ }^{1}$ Department of Paediatrics, Faculty of Medicine, University of Colombo, Sri Lanka and ${ }^{2}$ Children's Nutrition Research Centre, Department of Paediatrics and Child Health, University of Queensland, Brisbane, Australia.

Correspondence: VPW, e-mail <pujithaw@yahoo.com>. Received 22 April and revised version accepted 23 July 2009. Competing interests: none declared. 
Conclusions BMI is a poor indicator of the percentage fat and the commonly used cutoff values were not sensitive to detect cases of childhood obesity in Sri Lankan children.

\section{Introduction}

Worldwide incidence of non-communicable diseases is increasing. This is attributed to the increase in prevalence of obesity. Worldwide incidence of childhood obesity is estimated to be about $10 \%$ and in the AsiaPacific region is about 5\% [1]. In Sri Lanka the prevalence of overweight/obesity has increased and shows regional variation. A survey showed that the overall prevalence of overweight was about $2.2 \%$ with a sector variation [2]. Among urban school children overweight prevalence was $3-5 \%$ and among rural students it was $1.7 \%(\mathrm{p}<0.05)$. Among 8-12 year old school children in Colombo metropolitan area the prevalence of overweight/ obesity was between $14-15 \%$ [3].

There is a relationship between body mass index (BMI) and morbidity as well as mortality due to various obesity related diseases. However, it was shown that \% FM was more closely related to these metabolic derangements. Therefore obesity is being defined as the presence of excess body fat associated with morbidity [4]. However, the amount of body fat associated with morbidity in children is debatable and few studies have shown it to be $20-25 \%$ in boys and $30 \%$ in girls $[5,6]$.

In children, BMI changes with age and therefore a single cutoff value cannot be used. A BMI above 95th centile was considered as a cutoff limit for obesity [7]. The Centre for Disease Control /National Centre for Health Statistics (CDC/NCHS) and British Child Growth Foundation have developed population based gender specific BMI centile charts as well as BMI-Z scores. The International Obesity Task Force (IOTF), has developed a set of cutoff values [8]. These obesity cutoff values are based on population distribution of an anthropometric parameter rather than on a biological end point. Because of this the ability of BMI to diagnose obesity accurately has been questioned. IOTF cutoff values had a very low sensitivity in detecting obesity among Australian children of white Caucasian and Sri Lankan origin [9]. Diagnosis of childhood obesity, using a single definition, in a multiethnic community had been unsuccessful as the relationship between BMI and body fatness is influenced by ethnicity [10]. This resulted in a debate on whether to use population specific [11] BMI cutoff values or ethnicity specific [12] BMI cutoff values to diagnose obesity. This study was carried out to study the reliability of use of different BMI cutoff values in diagnosing obesity in a group of Sri Lankan children, whose south Asian origin is known to have a high body fat content with a low BMI [13].

\section{Methods}

Five to 15-year old healthy Sri Lankan children were recruited from schools in Colombo which had a fair representation of all socio-economic groups. Stratified random sampling based on age and gender was used to recruit subjects. A class from each grade from each school was randomly selected and students were invited to participate and they were given an appointment to come to the clinical laboratory of the University Paediatric Unit of the Lady Ridgeway Hospital for Children, Colombo for the examination. The study was carried out from September 2004 to April 2005 in which body composition of Sri Lankan children were assessed and some of the results had been published previously [14]. Both parents and children were informed about the procedure. Informed written consent from parents and assent from children were obtained. The Ethical Review Committees of University of Colombo and Lady Ridgeway Hospital for Children approved the study.

The height and weight were measured using standard protocols and described elsewhere [14]. BMI was calculated by weight/height ${ }^{2}\left(\mathrm{kgm}^{-2}\right)$. Waist circumference (WC) was measured in the horizontal plane at the level of mid point between lower costal margin and upper margin of iliac crest in the mid axillary line [15]. Hip circumference (HC) was measured while the subject stood erect with arms at the sides of the body and feet together at the level of maximum extension of the buttocks that can be seen. Waist-hip ratio (WHR) was calculated by WC/HC.

The total body water (TBW) was measured by isotope dilution method using deuterium in the form of water $\left(\mathrm{D}_{2} \mathrm{O}\right)$. The complete technique is described elsewhere [14]. The absolute fat mass (FM) was calculated subtracting FFM from weight based on two-compartment body composition model. Percentage FM is when FM was expressed as a fraction of body weight. Percentage FM associated with morbidity was considered to be $25 \%$ for boys and $30 \%$ for girls and was considered as criterion diagnosis of obesity. Apart from this, obesity was diagnosed based on BMI cutoffs described by IOTF, CDC/ NCHS and British Growth Standards BMI-Z scores. BMI changes with growth from infancy through childhood to adolescents. Therefore BMI-Z score is considered as a better tool for follow up [16]. A BMI above 95th centile for age and gender on CDC/NCHS BMI centile or a BMI-Z above +2 in British Standards was considered to be obese. Obesity was also diagnosed based on percentage FM centile charts (\% FM >98th centile) [17] and WC centile charts (WC $>98$ th centile) [15].

Validity and accuracy of BMI indicators in the diagnosis of obesity were evaluated by calculating sensitivity, specificity, positive predictive value and efficiency, relative to true obesity diagnosed by absolute $\% \mathrm{FM}$ (using $\mathrm{D}_{2} \mathrm{O}$ ), using a $2 \times 2$ table and method is described elsewhere [9].

Pearson product moment correlation was calculated between anthropometric measures and FM, \% FM to 
determine the association between them as the most suitable measure. Significance was considered at $\mathrm{p}<0.05$. Data were analysed using NCSS computer package for Windows.

\section{Results}

Data of 282 children (158 boys and 124 girls) were used for the final analysis. Table 1 shows some characteristics of the participants. The correlation coefficients (r) for BMI, BMI-Z and FM, \%FM and FMI were calculated. Similarly correlation coefficients for FMI, WC, HC, WHR and FM, \% FM for each sex group as well as for whole group were calculated (Table 2). The relationship between BMI and FM and BMI and FMI were high compared to the relationship between BMI and \% FM. Similar association was seen between BMI-Z and FM, \% FM and FMI. The relationship between WC, with FM was higher than with \% FM. All these associations were statistically significant $(\mathrm{p}<0.001)$. Association between WHR and FM as well as \% FM was poor. Anthropometric measures had a stronger association with FM than with $\%$ FM.

Table 3 shows the number of individuals diagnosed as obese based on 5 different criteria. Absolute fat percentage calculated by isotope dilution method based on 2-compartment model was used as the reference. A high proportion of girls (80\%) had \% FM that would be associated with adverse health outcomes (obese), compared to boys (45.5\%). The IOTF BMI based cutoff detected $8(6.4 \%)$ girls and $9(5.7 \%)$ boys as obese. Number of obese cases detected by CDC centile cutoff as well as BMI-Z score was little higher than the number detected by IOTF cutoff values (Table 3). However, using \% FM centile charts, a higher number was diagnosed as obese (142) and was quite close to the number (171) detected by criterion method. WC measures were able to diagnose more cases of obesity than BMI based cutoff values in this group of children.

Table 4 shows the results of validation of each method used in the diagnosis of obesity in Sri Lankan children. In both boys and girls, the BMI based cutoff had a very low sensitivity ranging from 8-23.6\%, but high specificity (100\%). The positive predictive value was $100 \%$ in both groups but efficiency was 60-65.2\% in boys and 26.6-33\% in girls. Diagnosis of obesity based on percentage FM had a high sensitivity (77.8\% in boys and $81.8 \%$ in girls) and specificity (94.2\% in boys and $100 \%$ in girls). The positive predictive value as well as efficiency was also high.

Table 1. Physical characteristics of the study population

\begin{tabular}{lcc}
\hline & Male & Female \\
\hline$N$ & 158 & 124 \\
\hline Age $(\mathrm{yrs})$ & $9.6 \pm 2.7$ & $10.1 \pm 2.8$ \\
Height $(\mathrm{cm})$ & $134.4 \pm 15.4$ & $138.5 \pm 15.3^{*}$ \\
Weight $(\mathrm{kg})$ & $31.2 \pm 12.5$ & $35.0 \pm 12.7^{*}$ \\
BMI $\left(\mathrm{kg} / \mathrm{m}^{2}\right)$ & $16.6 \pm 3.7$ & $17.7 \pm 3.9^{*}$ \\
FFM $(\mathrm{kg})$ & $22.3 \pm 7.4$ & $21.7 \pm 7.9$ \\
FM $(\mathrm{kg})$ & $8.9 \pm 6.6$ & $13.2 \pm 6.9^{*}$ \\
$\%$ FM & $26.2 \pm 10.6$ & $37.0 \pm 10.3^{*}$ \\
WC $(\mathrm{cm})$ & $64.1 \pm 10.5$ & $60.5 \pm 11.4^{*}$ \\
HC $(\mathrm{cm})$ & $74.2 \pm 11.9$ & $68.7 \pm 11.4^{*}$ \\
WHR & $0.87 \pm 0.07$ & $0.88 \pm 0.05$ \\
WC Z score & $0.10 \pm 1.7$ & $0.93 \pm 1.6$ \\
BMI Z score & $-0.5 \pm 1.8$ & $-0.16 \pm 1.6$ \\
& & \\
\hline
\end{tabular}

* $\mathrm{p}<0.05$ between Caucasians and Sri Lankans within the gender. 
Table 2. Correlation coefficient ( $\mathbf{r}$ )

\begin{tabular}{lccccccc}
\hline & \multicolumn{2}{c}{ Female } & \multicolumn{2}{c}{ Male } & \multicolumn{2}{c}{ Whole group } \\
\hline & $r$ & $p$ & $r$ & $p$ & $r$ & $p$ \\
\hline BMI versus FM & 0.82 & $<0.001$ & 0.87 & $<0.001$ & 0.84 & $<0.001$ \\
BMI versus \%FM & 0.32 & $<0.001$ & 0.68 & $<0.001$ & 0.52 & $<0.001$ \\
BMI versus FMI & 0.78 & $<0.001$ & 0.88 & $<0.001$ & 0.83 & $<0.001$ \\
& & & & & & \\
BMI-Z versus FM & 0.60 & $<0.001$ & 0.72 & $<0.001$ & 0.66 & $<0.001$ \\
BMI-Z versus \%FM & 0.32 & $<0.001$ & 0.66 & $<0.001$ & 0.51 & $<0.001$ \\
BMI-Z versus FMI & 0.70 & $<0.001$ & 0.80 & $<0.001$ & 0.74 & $<0.001$ \\
& & & & & & \\
FMI versus FM & 0.92 & $<0.001$ & 0.95 & $<0.001$ & 0.94 & $<0.001$ \\
FMI versus \%FM & 0.83 & $<0.001$ & 0.93 & $<0.001$ & 0.90 & $<0.001$ \\
& & & & & & \\
WC versus FM & 0.80 & $<0.001$ & 0.89 & $<0.001$ & 0.85 & $<0.001$ \\
WC versus \%FM & 0.31 & $<0.001$ & 0.66 & $<0.001$ & 0.52 & $<0.001$ \\
& & & & & & \\
WC-Z versus FM & 0.58 & $<0.001$ & 0.72 & $<0.001$ & 0.68 & $<0.001$ \\
WC-Z versus \%FM & 0.29 & $<0.001$ & 0.66 & $<0.001$ & 0.54 & $<0.001$ \\
& & & & & & \\
HC versus FM & 0.79 & $<0.001$ & 0.87 & $<0.001$ & 0.84 & $<0.001$ \\
HC versus \%FM & 0.21 & $<0.001$ & 0.61 & $<0.001$ & 0.47 & $<0.001$ \\
WHR versus FM & & & & & & \\
WHR versus \%FM & 0.04 & 0.660 & 0.34 & $<0.001$ & 0.14 & 0.017 \\
& 0.21 & 0.020 & 0.36 & $<0.001$ & 0.20 & $<0.001$ \\
\hline
\end{tabular}

Table 3. Cases of obesity and overweight diagnosed by different parameters

\begin{tabular}{lcc}
\hline & $\begin{array}{c}\text { Female } \\
(124)\end{array}$ & $\begin{array}{c}\text { Male } \\
(158)\end{array}$ \\
\cline { 2 - 3 } Obesity by & $n(\%)$ & $n(\%)$ \\
\hline \%FM & $99(80.0)$ & $72(45.5)$ \\
IOTF & $8(6.4)$ & $9(5.7)$ \\
CDC & $16(13.0)$ & $14(8.6)$ \\
BMI-Z>2SD & $16(13.0)$ & $17(10.7)$ \\
\%FM centile & $81(65.0)$ & $61(38.6)$ \\
WC & $38(30.6)$ & $26(16.4)$ \\
\hline
\end{tabular}


Table 4. Validity of BMI as an indicator of obesity (\%)

\begin{tabular}{|c|c|c|c|c|c|c|}
\hline & & $\begin{array}{l}\text { IOTF } \\
(\%)\end{array}$ & $\begin{array}{l}C D C \\
(\%)\end{array}$ & $\begin{array}{c}B M I- \\
Z>2 S D(\%)\end{array}$ & $\begin{array}{l}\% F M>98 t h \\
\text { Centile (\%) }\end{array}$ & $\begin{array}{c}W C>98 \text { th } \\
\text { Centile (\%) }\end{array}$ \\
\hline \multirow[t]{4}{*}{ Male } & Se & 12.5 & 19.5 & 23.6 & 77.8 & 34.7 \\
\hline & $\mathrm{Sp}$ & 100.0 & 100.0 & 100.0 & 94.2 & 98.8 \\
\hline & $\mathrm{Pv}$ & 100.0 & 100.0 & 100.0 & 91.8 & 96.0 \\
\hline & $\mathrm{Ef}$ & 60.0 & 65.2 & 65.2 & 86.7 & 69.6 \\
\hline \multirow[t]{4}{*}{ Female } & Se & 8.0 & 16.2 & 16.2 & 81.8 & 38.4 \\
\hline & Sp & 100.0 & 100.0 & 100.0 & 100.0 & 100.0 \\
\hline & $\mathrm{Pv}$ & 100.0 & 100.0 & 100.0 & 100.0 & 100.0 \\
\hline & $\mathrm{Ef}$ & 26.6 & 33.0 & 33.0 & 85.5 & 50.8 \\
\hline
\end{tabular}

Se - Sensitivity, Sp - Specificity, Pv - Predictive value, Ef - Efficiency

\section{Discussion}

Many changes in the body that occur during childhood in relation to non communicable disease persist into adulthood. Fatness in childhood and adolescence persists into adulthood and it strengthens with increase in age. Although the risk is less in younger age groups, obesity diagnosed at 7 years will have an elevated relative risk, of about 3, of becoming obese in adulthood [18].

Although exact amount is not known, the fat content of the body is the main risk factor for the development of obesity related morbidity. Therefore the best method to diagnose obesity would be to measure the FM directly. However, as this is not easy and practical in a busy clinic setting or epidemiological studies BMI was used as a surrogate measure. BMI, which is a ratio between weight and height, does not always reflect the percentage body fat accurately. In this study we saw a weak association between BMI and \%FM. Therefore its clinical relevance is doubtful. However, its simplicity and reproducibility has made it the most popular diagnostic tool of obesity.

IOTF cutoff values underestimate the prevalence of obesity $[9,19]$. A drawback of IOTF definition is that although it has a large data set from six countries, it did not have a fair representation from Asia. Therefore whether IOTF cutoff values are truly international and could be applied universally is of doubt.

WHO technical report [4] acknowledged that BMI cutoff values for overweight and obesity may not correspond to the same degree of fat content across different populations. Therefore population specific rather than universal BMI cutoff values should be used [20]. It appears that the population distribution of BMI and \%FM does not run parallel to each other in this study group as seen among white Caucasians. This shows that ethnicity influences body composition and south Asian children have a high fat content at a low BMI. BMI is an easy tool to use to diagnose obesity in community. However, in view of its low sensitivity in diagnosing obesity, best would be either to revise BMI cutoff values to suit a population/ ethnic group or make a definitive assessment of fat content of body and diagnose obesity based on that.

In this study the diagnosis of obesity was based on \%FM cutoff values defined on western populations. These \%FM associated with morbidity may not be accurate for Sri Lankan children. The body fat content that would be associated with morbidity in Sri Lankan children needs to be defined. As ethnicity has an influence in body composition, ideal would be to define local standards for screening and diagnosis of obesity.

\section{Acknowledgements}

We are grateful to Dr. Samanmali Sumanasena for collecting the data. This study was carried out with the financial support from Children's Nutrition Research Centre, Department of Paediatrics and Child Health, University of Queensland, Brisbane, Australia.

\section{References}

1. Lobstein T, Baur L, Uauy R. Obesity in children and young people: a crisis in public health. Obesity Reviews 2004; 5 : 4-85.

2. Jayatissa R, Ranbanda RM. Prevalence of challenging nutritional problems among adolescents in Sri Lanka. Food and Nutrition Bulletin 2006: 27: 153-60.

3. Wickramasinghe VP, Lamabadusuriya SP, Atapattu N, et al. Nutritional status of schoolchildren in an urban area of Sri Lanka. The Ceylon Medical Journal 2004; 49: 114-8.

4. WHO Technical Report Series - 894. Obesity: preventing and managing the global epidemic. WHO, Geneva. 2000.

5. Lohman TG. The prevalence of obesity in children in the United States. In: Advances in body composition assessment. Human Kinetics, Champaign IL. Monogram 1992; 3: 79-89. 
6 Dwyer T, Blizzard CL. Defining obesity in children by biological endpoint rather than population distribution. International Journal of Obesity 1996; 20: 472-80.

7 Barlow SE, Dietz WH. Obesity evaluation and treatment: expert committee recommendations. Paediatrics, 1998; 102: (http://www.pediatrics.org/cgi/content/full/102/3/e29. Accessed on 13th January 2002)

8 Cole TJ, Bellizzi MC, Flegal KM, Dietz WH. Establishing a standard definition for child overweight and obesity world wide: international survey. British Medical Journal 2000; 320: $1-6$.

9 Wickramasinghe VP, Cleghorn GJ, Edmiston KA, et al. Validity of BMI as a measure of obesity in Australian white Caucasian and Australian Sri Lankan children. Annals of Human Biology 2005; 32: 60-72.

10 Rush EC, Puniani K, Valencia ME, et al. Estimation of body fatness from body mass index and bioelectrical impedance: comparison of New Zealand European, Maori and Pacific Island children. European Journal of Clinical Nutrition 2003; 57: 1394-401.

11. Stevens J. Ethnic-specific revisions of body mass index cutoffs to define over weight and obesity in Asians are not warranted. International Journal of Obesity 2003; 27: 1297-9.

12. Misra A. Revisions of cutoffs of body mass index to define overweight and obesity are needed for the Asian ethnic groups. International Journal of Obesity 2003; 27: 1294-6.

13. Wang J, Thornton JC, Russel M, et al. Asians have lower body mass index (BMI) but higher percent body fat than do whites: comparisons of anthropometric measurements. American Journal of Clinical Nutrition 1994; 60: 23-8.

14. Wickramasinghe VP, Lamabadusuriya SP, Cleghorn GJ, Davies PSW. Assessment of body composition in Sri Lankan children: validation of a skin fold thickness equation. The Ceylon Medical Journal 2008; 53: 83-8.

15. McCarthy HD, Jarrett KV, Crawley HF. The development of waist circumference percentiles in British children aged 5.0-16.9 yrs. European Journal of Clinical Nutrition 2001; 55: $902-7$

16. Reinehr T, Kiess W, Andler W. Insulin sensitivity indices of glucose and free fatty acid metabolism in obese children and adolescents in relation to serum lipids. Metabolism 2005; 54: 397-402.

17. McCarthy HD, Cole TJ, Fry T, et al. Body fat reference curves for children. International Journal of Obesity Research 2006; 30: 598-602.

18. Power C, Lake JK, Cole TJ. Measurement and long term health risks of child and adolescent fatness. International Journal of Obesity 1997; 21: 507-26.

19. Flegal KM, Ogden CL, Wei R, et al. Prevalence of overweight in US children: comparison of US growth charts from the centres for disease control and prevention with other reference values for body mass index. American Journal of Clinical Nutrition 2001; 73: 1086-93.

20. Deurenberg P, Yap M, Van Staveren WA. Body mass index and percentage body fat: a meta analysis among different ethnic groups. International Journal of Obesity 1998; 22: 1164-71. 